\title{
Bose-Einstein condensation in semiconductors: myth or reality?
}

Jacek Kasprzak

kasprzakj@Cardiff.ac.uk

Maxime Richard

maxime.richard@grenoble.cnrs.fr

Régis André

regis.andre@grenoble.cnrs.fr

Le Si Dang

lesidang@grenoble.cnrs.fr

\author{
CEA-CNRS-UJF joint group "Nanophysique et Semiconducteurs", Institut Néel, CNRS-UJF, 38042 \\ Grenoble, France \\ Ecole Polytechnique Fdrale de Lausanne (EPFL), Station 3, CH-1015 Lausanne, Switzerland
}

CEA-CNRS-UJF joint group “Nanophysique et Semiconducteurs", Institut Néel, CNRS-UJF, 38042 Grenoble, France

CEA-CNRS-UJF joint group "Nanophysique et Semiconducteurs", Institut Néel, CNRS-UJF, 38042 Grenoble, France

Bose-Einstein condensation, predicted for a gas of non interacting bosons in 1924 by Einstein, has been demonstrated for the first time in 1995 in a dilute gas of rubidium atoms at temperatures below $10^{-6} \mathrm{~K}$. In this work, it is shown that Bose-Einstein condensation can be achieved at around $15-20 \mathrm{~K}$ in a solid state system by using microcavity polaritons, which are composite bosons of mass ten billion times lighter than that of rubidium atoms. [DOI: 10.2971/jeos.2008.08023]

Keywords: Bose-Einstein condensation, microcavity polaritons, excitons

\section{INTRODUCTION}

Particles in nature can be divided into two categories, depending on their spin: those with integer spins are bosons, and those with half-integer spins are fermions. A specific feature of bosons is that they can occupy the same quantum state, and undergo the so-called Bose-Einstein condensation (BEC) when their thermal de Broglie wavelength becomes comparable to their average separation. Then a large fraction of bosons in the system would condense in the lowest quantum state, resulting in the appearance of macroscopic coherence. Since the de Broglie wavelength scales as the inverse of the square root of the particle mass and temperature, the BEC criterion is most easily satisfied at high temperature for bosons with a light mass.

BEC was demonstrated for the first time in a dilute gas of rubidium atoms in 1995. Because of the heavy atom mass (about five orders of magnitude larger than the electron mass), sophisticated cooling techniques were needed to lower the atomic gas temperature down to the micro-Kelvin range to achieve condensation [1]. For solid state systems, excitons in semiconductors have long been considered as a promising candidate for BEC [2]. A semiconductor is characterized by the existence of an energy band gap separating the valence band, i.e. the uppermost energy band full of electrons at low temperature, from the conduction band. Electrons in the valence band can be excited into the conduction band (by optical excitation for example), leaving behind unoccupied states in the valence band or "hole" states. The associated quasiparticles are called holes, behaving as positively charged particles, and thus can be bound to electrons by Coulomb interaction, forming positronium-like particles called excitons. Al- though electrons and holes are fermions, excitons are (composite) bosons. Moreover their light mass, of the order of the electron mass, would permit BEC at temperatures of a few Kelvin, reachable by standard cryogenic techniques. However, in spite of intense research efforts over the past three decades, no convincing evidence of exciton condensation has ever been firmly established.

\section{WHY MICROCAVITY POLARITONS?}

Recently new directions have been explored for BEC in semiconductors, using two-dimensional (2D) quantum structures, such as coupled quantum wells under an external applied electric field or quantum wells embedded in optical microcavities [2]. The second system consists in planar Fabry-Perot resonators whose optical length is tuned to a half-integer multiple of the emission wavelength of quantum well excitons. The strong light-matter interaction between cavity photons and excitons leads to the formation of new eigenstates (see Figure 1) called polaritons, which are half-light, half-matter bosonic quasi-particles [3]. The extremely steep dispersion of the cavity polariton modes, due to the optical confinement along the cavity length, results in a typical polariton effective mass of about $5 \times 10^{-5}$ times the free electron mass. The corresponding de Broglie wavelength would be around $2 \mu \mathrm{m}$ at $20 \mathrm{~K}$, so that the BEC criterion could be met with a polariton density as low as $N=10^{8} \mathrm{~cm}^{-2}$.

From the experimental side, we note that the polariton distribution in the momentum space can be conveniently probed by analysing the far-field emission, because the light emitted 


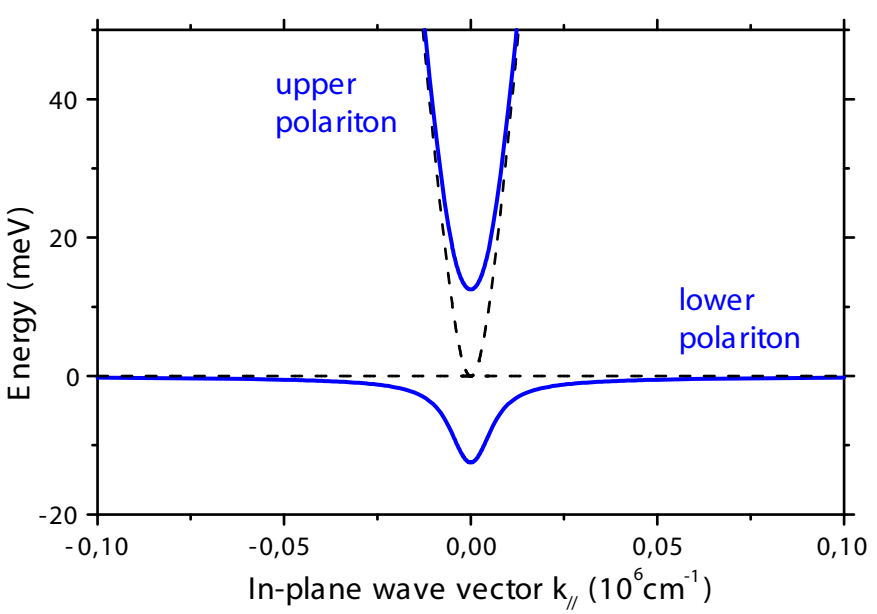

FIG. 1 Polariton energy dispersion as a function of the in-plane wavevector $k_{/ /}$, calculated for the 16 quantum well CdTe microcavity used in this work. The origin of energy is that of exciton emission in quantum wells at $1685 \mathrm{meV}$. Interaction between exciton and photon modes (dashed curves) gives rise to lower and upper polariton branches (solid curves) with dispersions featuring an anticrossing, typical of the strong coupling regime. Polaritons with $k_{/ /} \sim 0$ in the lower polariton branch are good candidates for Bose-Einstein condensation since their mass is about five orders of magnitude lighter than the electron mass. The radiative recombination of polaritons results in the emission of photons that can be used to probe the population distribution in the lower polariton branch. Photons emitted at angle $\theta$ correspond to polaritons of energy $E$ and in-plane wavevector $k_{/ /}=E /(\hbar c) \sin \theta$.

by the microcavity is part of the polariton wavefunction. On the other hand, the drawback of the strong photon-exciton coupling is the very short polariton lifetime, typically around $10^{-12} \mathrm{~s}$, which could be an obstacle to reach thermal equilibrium with the host lattice. However, it will be shown below that Coulomb scatterings under high polariton density are fast enough to achieve internal equilibration within the exciton polariton gas. Strictly speaking, polariton condensation, which eventually occurs, is not the thermodynamic phase transition discussed by Einstein, but an out-of-equilibrium phase transition. Nevertheless, it displays all the defining BEC features, such as massive occupation of the ground state, macroscopic spatial coherence and temporal coherence.

\section{THERMALIZATION AND CONDENSATION}

To demonstrate BEC, a CdTe-based microcavity embedding 16 quantum wells has been grown by Molecular Beam Epitaxy. Hot electron-hole pairs are injected in the microcavity by continuous wave laser pumping. Excitons are formed and relax through exciton-phonon and exciton-exciton scatterings. They strongly interact with the cavity photon modes to form polaritons. The distribution of these polaritons in the EnergyMomentum space $\left(E, k_{/ /}\right)$can be probed by measuring the microcavity far-field emission [4]. Figure 2 shows 2D images of the polariton distribution on the lower polariton branch, measured at $T=5 \mathrm{~K}$, for three pumping powers. At low pumping power (left panel), polaritons are smoothly distributed along their parabolic energy dispersion curve, over a broad range of $E$ and $k_{/ /}$. However, above some critical power $P_{0}$ (middle and right panels), condensation occurs, evidenced by the massive occupation of the $k_{/ /}=0$ ground state, whereas the excited state occupation remains almost unchanged. This bimodal distribution is typical of BEC at finite temperature.

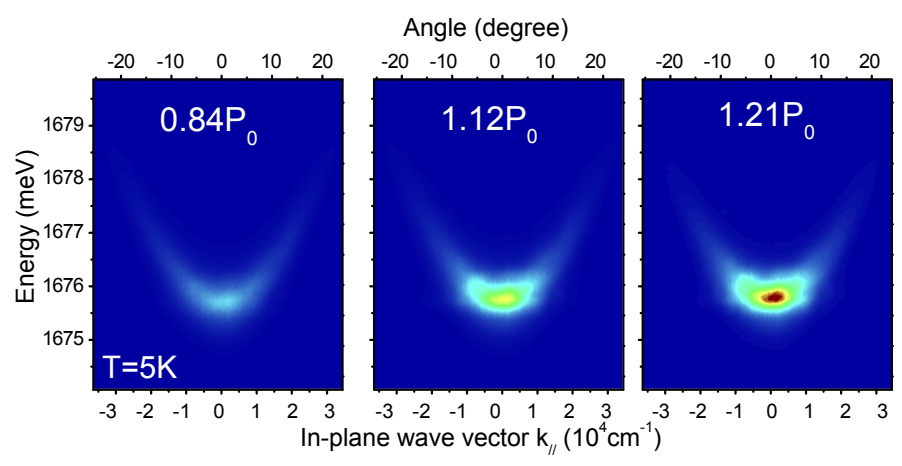

FIG. 2 (Energy $E$, momentum $k_{/ /}$) images displaying population distribution in the lower polariton branch measured at $T=5 \mathrm{~K}$, for three laser pumping powers: 0.84 , 1.12 , and $1.21 P_{0}$, where $P_{0}$ is the condensation threshold power. The bimodal distribution, consisting of massively occupied ground state and thermal cloud in the excited states, clearly emerges above threshold.

Condensation can be driven also by the temperature. Figure 3 displays $2 \mathrm{D}$ images of the polariton distribution measured for the same pumping power but for different lattice temperatures. The bimodal distribution would appear only (right panel) when the temperature is below the critical value of $25 \mathrm{~K}$ in this particular experiment.

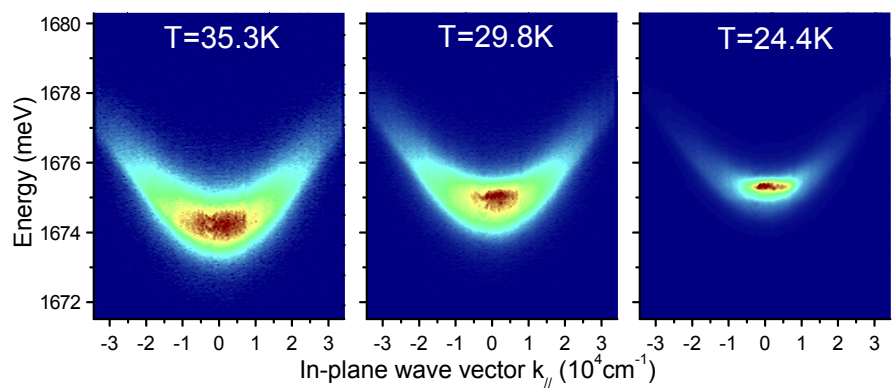

FIG. 3 (Energy $E$, momentum $k_{/ /}$) images displaying population distribution in the lower polariton branch measured for the same laser pumping power, but for three lattice temperatures. Polariton condensation is evidenced by the onset of the bimodal distribution at $T=24.4 \mathrm{~K}$.

Data such as those shown in Figure 2 can be used to extract the occupation of polariton states as a function of their energy, and results are displayed in Figure 4 for various pumping powers. The bimodal distribution can be clearly observed when pumping above some threshold power $P_{0}$. The thermal cloud of excited polaritons follows the Maxwell-Boltzmann distribution with an effective temperature of $T=16 \mathrm{~K}$. In Figure 4 , it should be noted that the polariton temperature and occupation of excited levels do not change much with increasing pumping above threshold. It indicates that, above the critical density, any polaritons added in the system would condense into the ground state, as expected for BEC.

At first sight, the observation of a polariton gas in internal thermal equilibrium could appear puzzling considering the short polariton radiative lifetime. In fact, analysis of polariton dephasing shows that the polariton-polariton scattering time at pumping around threshold is shorter than the polariton life- 


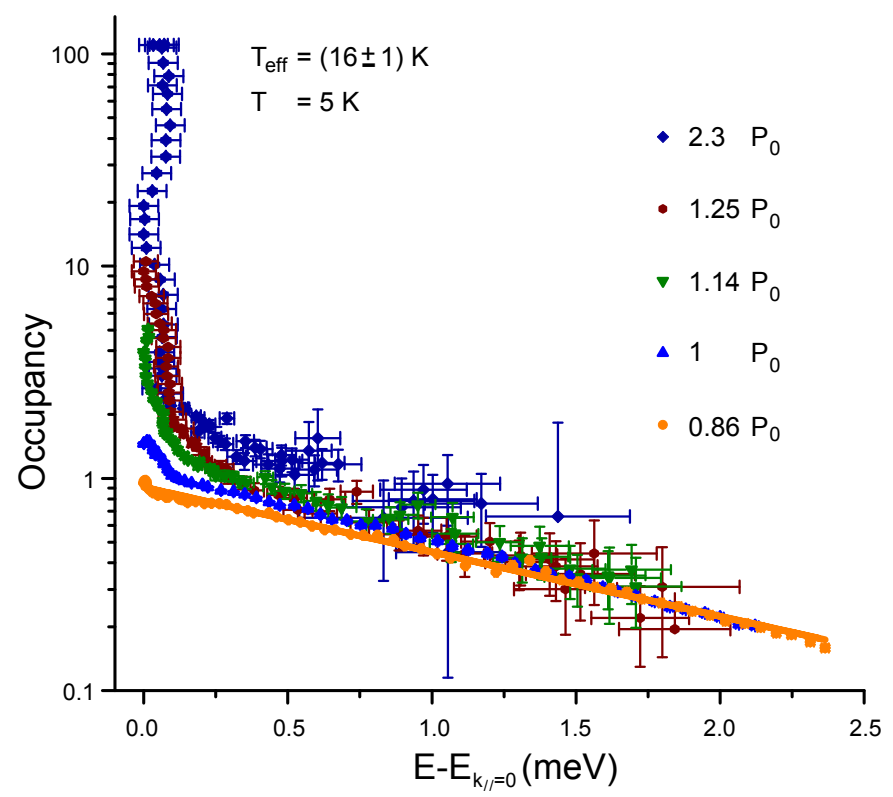

FIG. 4 Occupation (on a logarithmic scale) as a function of the polariton energy and the pumping power, measured at $T=5 \mathrm{~K}$. For pumping just below threshold, the population occupation can be fitted with a Maxwell-Boltzmann distribution and an effective temperature of $T_{\text {eff }}=16 \mathrm{~K}$. Above threshold, the ground state is massively occupied, by contrast to the saturation effect observed for the excited states.

time by a factor 2 (not shown), which would allow thermal equilibration within the polariton gas before escape of polaritons out of the microcavity.

In [4], the long range order expected for BEC has been investigated by measuring the first order spatial correlation function within the emitting spot, using a Michelson interferometer. For pumping below threshold, no spatial correlation can be found beyond the polariton de Broglie wavelength (about $2 \mu \mathrm{m}$ at $T=20 \mathrm{~K})$. Above threshold however, correlation extends across the whole emitting spot of $20 \mu \mathrm{m}$ diameter, evidencing the long range spatial coherence of the condensate. First and second order temporal correlation functions have been measured also. Results fully support the onset of coherence at condensation threshold and the quantum nature of the condensed polariton gas $[4,5]$.

Finally, quantized vortices, expected for BEC systems of strongly interacting bosons, have been recently reported in a CdTe polariton condensate [6]. However, by contrast to superfluid helium and atomic BEC gases, these vortices result from the combination of two polariton features, i.e. the pump-and-decay nature of the polariton gas and disorder in the microcavity.

\section{OUTLOOK}

We have shown that exciton polaritons can be condensed in semiconductors, in spite of their very short lifetime, typically one picosecond. In $\mathrm{CdTe}$, the condensation threshold at lattice temperature of $5 \mathrm{~K}$ is found to be more than two orders of magnitude lower than the standard lasing threshold. Thus polariton condensates could be used as a low threshold source of coherent photons (polariton "laser"). To bring the critical temperature closer to room temperature, one could use wider band gap semiconductors, such as $\mathrm{GaN}$ or $\mathrm{ZnO}$, to benefit from their higher exciton stability under high pumping power at high temperature.

\section{References}

[1] See for example: http://jilawww.colorado.edu/bec/ and http://cua. mit.edu/ketterle_group/

[2] For a review of BEC research on exciton systems, see: D. Snoke, "Spontaneous Bose coherence of excitons and polaritons" Science 298, 1368 (2002).

[3] A. Kavokin, and G. Malpuech, Cavity polaritons (Elsevier, Amsterdam, 2003).

[4] J. Kasprzak, M. Richard, S. Kundermann, A. Baas, P. Jeambrun, J. M. J. Keeling, F. M. Marchetti, M. H. Szymańska, R. André, J. L. Staehli, V. Savona, P. B. Littlewood, B. Deveaud, and Le Si Dang, "Bose-Einstein condensation of exciton polaritons" Nature 443 , 409-414 (2006).

[5] J. Kasprzak, M. Richard, A. Baas, B. Deveaud, R. Andre, J.-Ph. Poizat, and Le Si Dang, "Second-Order Time Correlations within a Polariton Bose-Einstein Condensate in a CdTe Microcavity" Phys. Rev. Lett. 100, 067402 (2008).

[6] K. G. Lagoudakis, M. Wouters, M. Richard, A. Baas, I. Carusotto, R. André, Le Si Dang, and B. Deveaud-Pledran, "Quantised Vortices in an Exciton-Polariton Fluid" arXiv:0801.1916V1 [cond-mat.other] (2008). 